\title{
An immune-related IncRNA signature as a prognostic immunotherapy target for colon cancer
}

\author{
Bin Wu ( 2017203020081@whu.edu.cn ) \\ Wuhan University Renmin Hospital https://orcid.org/0000-0003-0565-0267 \\ Yi Yao \\ Wuhan University Renmin Hospital \\ Yi Dong \\ Wuhan University Renmin Hospital \\ Si Qi Yang \\ Wuhan University Renmin Hospital \\ Deng Jing Zhou \\ Wuhan University Renmin Hospital \\ Meng Xia Xiao \\ Wuhan University Renmin Hospital \\ Qi Bin Song \\ Wuhan University Renmin Hospital
}

Primary research

Keywords: colon cancer; IncRNA; immune; survival analysis

Posted Date: July 20th, 2020

DOl: https://doi.org/10.21203/rs.3.rs-35120/v2

License: () (1) This work is licensed under a Creative Commons Attribution 4.0 International License.

Read Full License 
The authors have withdrawn this preprint from Research Square 